\title{
Optimal recovery of derivatives of Hardy class functions
}

\author{
Mikhail Ovchintsev ${ }^{1, *}$ \\ ${ }^{1}$ Moscow State University of Civil Engineering, Yaroslavskoye shosse, 26, Moscow, 129337, Russia
}

\begin{abstract}
The paper considers the best linear method for approximating the values of derivatives of Hardy class functions in the unit circle at zero according to the information about the values of functions at a finite number of points $z_{1}, \ldots, z_{n}$ that form a regular polygon, and also the error of the best method is obtained. The introduction provides the necessary concepts and results from the papers of K.Yu. Osipenko. Some results of the studies of S.Ya. Khavinson and other authors are also mentioned here. The main section consists of two parts. In the first part of the second section, the research method is disclosed, namely, the error of the best method for approximating the derivatives at zero according to the information about the values of functions at the points $z_{1}, \ldots, z_{n}$ is calculated; the corresponding extremal function is written out. It is established that for $p>1$, the corresponding extremal function is unique up to a constant factor that is equal to one in modulus. For $p=1$, the corresponding extremal function is not unique. All such corresponding extremal functions are determined here. In the second part of the second section, it is proved that for all $\mathrm{p}(1 \leq p<$ $\infty$ ), the best linear approximation method is unique, and the coefficients of the best linear recovery method are calculated. The expressions used to calculate the coefficients are greatly simplified. At the end of the paper, the obtained results are described, and possible areas for further research are indicated.
\end{abstract}

\section{Introduction}

The problems of optimal recovery of derivatives of analytic functions of the Hardy class, given in the unit circle in accordance with the information about their values in a finite number of points, have been considered in many papers (see [1] - [5]). In this paper, a particular case is considered, namely, the values of $f^{\prime}(0)$ are optimally recovered by the values of $f\left(z_{1}\right), \ldots, f\left(z_{n}\right)$, where the points $z_{1}, \ldots, z_{n}$ form a regular polygon $\left(f(z) \in H^{p}, 1 \leq\right.$ $p<\infty)$. In this case, the coefficients of the best linear method that were found as a result of the recovery have a very simple form.

Let's recall some concepts and results from [6]. Let $\mathrm{W}$ be some set lying in the linear space $\mathrm{X}$ and $L, l_{1}, \ldots, l_{n}$ are linear functionals defined in $\mathrm{X}$. If $S\left(t_{1}, \ldots, t_{n}\right)$ is any complex function $\mathrm{n}$ of complex variables, then the approximation error of the method $\mathrm{S}$ is called the following value

\footnotetext{
*Corresponding author:6714543@rambler.ru
} 


$$
r_{n}(S)=\sup _{x \in W}\left|L(x)-S\left(l_{1}(x), \ldots, l_{n}(x)\right)\right| .
$$

The method $S_{0}\left(t_{1}, \ldots, t_{n}\right)$ is called the best approximation method if

$$
r_{n}\left(S_{0}\right)=\inf _{S} r_{n}(S)
$$

In [6], the existence of the best linear approximation method $S_{0}=\sum_{k=1}^{n} c_{k} l_{k}(x)$ was proved (under certain conditions at $\mathrm{W}$ ) (here $c_{k}$ - some complex numbers). Moreover, the error of the best approximation method can be found by the formula

$$
r_{n}\left(S_{0}\right)=\sup _{\substack{x \in W \\ l_{1}(x)=. .=l_{n}(x)=0}}|L(x)| .
$$

Let $K=\{z:|z|<1\}-$ unit circle, and $\Gamma=\{|z|=1\}-$ unit circumference. Let's denote

$$
H_{1}^{p}=\left\{f(z), f(z) \in H^{p}: \int_{\Gamma}|f(\zeta)|^{p} d \varphi \leq 1\right\}-
$$

unit ball in the space $H^{p}$ (for the definition of the $H^{p}$ classes of analytic functions in $\mathrm{K}$, see [7], [8]). Let us introduce the following points that form a regular polygon

$$
z_{1}=R, z_{2}=R e^{i \frac{2 \pi}{n}}, \ldots, z_{n}=R e^{i(n-1) \frac{2 \pi}{n}},(2)
$$

where $R-$ some number $(0<R<1 ; n \geq 3)$. If we consider that

$$
L(f)=f^{\prime}(0), l_{1}(f)=f\left(z_{1}\right), \ldots, l_{n}(f)=f\left(z_{n}\right),
$$

then the error of the best method of approximating the values of $f^{\prime}(0)$ by the values of $f\left(z_{1}\right), \ldots, f\left(z_{n}\right)$ is found by the formula (let's denote by $r_{1, p}\left(0, z_{1}, \ldots, z_{n}\right)$; see (1), (3))

$$
r_{1, p}\left(0, z_{1}, \ldots, z_{n}\right)=\sup _{\substack{f(z) \in H_{p}^{1} \\ f\left(z_{1}\right)=. .=f\left(z_{n}\right)=0}}\left|f^{\prime}(0)\right| .
$$

Now let us present some results from [9], [10]. If $\omega(\zeta)$ is a bounded function on the circumference $\Gamma$, then

$$
\sup _{f \in H_{1}^{1}}\left|\int_{\Gamma} f(\zeta) \omega(\zeta) d \zeta\right|=\min _{\varphi \in B(K)} \operatorname{vraimax}|\omega(\zeta)-\varphi(\zeta)|,
$$

where $B(K)$ - the space of bounded, analytic in $\mathrm{K}$ functions. In order for the functions $\mathrm{f} f^{*}(z) \in H_{1}^{1}$ and $\varphi^{*}(z) \in B(K)$ to be extreme in the equality (5), it is necessary and sufficient that the following relation would be satisfied almost everywhere on $\Gamma$

$$
f^{*}(\zeta)\left[\omega(\zeta)-\varphi^{*}(\zeta)\right] d \zeta=e^{i \alpha} \lambda\left|f^{*}(\zeta)\right| d \varphi
$$

( $\alpha$ real, $\lambda-$ the total value of both extrema in (5)).

Let $p>1$. Then if $\omega(\zeta) \in L_{q}(\Gamma)\left(\frac{1}{p}+\frac{1}{q}=1\right)$, then

$$
\sup _{f \in H_{p}^{1}}\left|\int_{\Gamma} f(\zeta) \omega(\zeta) d \zeta\right|=\min _{\varphi \in H_{q}}\left\{\int_{\Gamma}|\omega(\zeta)-\varphi(\zeta)|^{q} d \varphi\right\}^{\frac{1}{q}}
$$

The extremal function $\varphi^{*}(\zeta)$ is unique in the right sides of equalities (5) and (7). For $p>$ 1 , the extremal function $f^{*}(z)$ on the left side of the equality (7) is unique up to a constant factor $e^{i \delta}(\delta \in R)$. In order for the functions $f^{*}(z) \in H_{p}^{1}$ and $\varphi^{*} \in H_{q}$ to be extremal in the 
equality (7), it is necessary and sufficient that the following relation would be satisfied almost everywhere on $\Gamma$

$$
f^{*}(\zeta)\left[\omega(\zeta)-\varphi^{*}(\zeta)\right] d \zeta=e^{i \alpha} \lambda\left|f^{*}(\zeta)\right|^{p} d \varphi,
$$

where $\lambda-$ the total value in the equality (7), $\alpha \in R$. It is also known that if $f^{*}(z)-$ the extremal function on the left side of the equality (7) (or (5)), then

$$
\int_{\Gamma}\left|f^{*}(\zeta)\right|^{p} d \varphi=1
$$

And finally, if $\omega(\zeta)$ is a boundary value on $\Gamma$ of a $\omega(z)$ function meromorphic in $\mathrm{K}$ with poles $\beta_{1}, \ldots, \beta_{m}$ (each pole is repeated as many times as its multiplicity), then the product

$$
R(z)=f^{*}(z)\left[\omega(z)-\varphi^{*}(z)\right]
$$

is analytical on $\Gamma$ and has in $\mathrm{K}$

$$
t=m-1
$$

zeros. In the papers [9], [10], the following is also established (see (10), (11))

$$
R(z)=C \frac{\prod_{k=1}^{m-1}\left(z-\alpha_{k}\right)\left(1-\overline{\alpha_{k}} z\right)}{\prod_{k=1}^{m}\left(z-\beta_{k}\right)\left(1-\overline{\beta_{k}} z\right)}
$$

where $\left|\alpha_{k}\right| \leq 1, k=1, \ldots, m-1 ; C-$ constant number.

Note that this paper is a continuation of [11], in which the coefficients of the best linear method for approximating the values of $f(0)$ by the values of $f\left(z_{1}\right), \ldots, f\left(z_{n}\right)$, where $f(z) \in$ $B(K)$, are found. We need the following equality below (see [3])

$$
\sup _{F(z) \in H_{p}^{1}}|F(a)|=\frac{1}{(2 \pi)^{\frac{1}{p}}\left(1-|a|^{2}\right)^{\frac{1}{p}}}
$$

where $a-$ given number $(|a|<1)$. It was proved in [9] that the extremal function $F^{*}(z)$ of the problem (13) is unique up to a constant factor $e^{i \delta}, \delta \in R$ and has the form

$$
F^{*}(z)=e^{i \delta} \frac{\left(1-|a|^{2}\right)^{\frac{1}{p}}}{(2 \pi)^{\frac{1}{p}}(1-\bar{a} z)^{\frac{2}{p}}}
$$

with all $\mathrm{p}(1 \leq p<\infty)$. And finally, in the future, we will use the formulas (see [11])

$$
\begin{aligned}
& \prod_{j=2}^{n}\left(R-z_{j}\right)=R^{n-1} n, \\
& \prod_{j=2}^{n}\left(1-\bar{z}_{j} R\right)=\frac{1-R^{2 n}}{1-R^{2}},
\end{aligned}
$$

where points $z_{1}, \ldots, z_{n}$ form a regular polygon (see (2)).

\section{Methods}

\subsection{Finding the error of the best method of approximating the values of $\boldsymbol{f}^{\prime}(0)$ by the values of $f\left(z_{1}\right), \ldots, f\left(z_{n}\right)$, where $f(z) \in H_{p}$}

Let's denote a finite Blaschke product by

$$
B(z)=\prod_{k=1}^{n} \frac{z-z_{k}}{1-\overline{z_{k}} z} .
$$


Lemma 1. In the case when the points $z_{1}, \ldots, z_{n} \quad(n \geq 3)$ have the form (2), a finite Blaschke product has the following properties:

$$
\begin{gathered}
B(z)=B\left(e^{i \frac{2 \pi}{n}} z\right), \\
B(0)=-R^{n}, \\
B^{(j)}(0)=0,
\end{gathered}
$$

when $1 \leq j \leq n-1$.

Proof. First, we prove the equality (18). Indeed,

$$
\begin{aligned}
& B\left(e^{i \frac{2 \pi}{n}} Z\right)=\frac{e^{i \frac{2 \pi}{n}} Z-R}{1-R e^{i \frac{2 \pi}{n}} Z} \frac{e^{i \frac{2 \pi}{n}} Z-R e^{i \frac{2 \pi}{n}}}{1-R e^{-i \frac{2 \pi}{n}} e^{i \frac{2 \pi}{n}} Z} \ldots \frac{e^{i \frac{2 \pi}{n}} Z-R e^{i(n-1) \frac{2 \pi}{n}}}{1-R e^{-i(n-1) \frac{2 \pi}{n}} e^{i \frac{2 \pi}{n}} Z} \\
& =\frac{Z-R e^{-i \frac{2 \pi}{n}}}{1-R e^{i \frac{2 \pi}{n}} Z} \frac{z-R}{1-R Z} \ldots \frac{z-R e^{i(n-2) \frac{2 \pi}{n}}}{1-R e^{-i(n-2) \frac{2 \pi}{n}} Z}=B(z) .
\end{aligned}
$$

Let us prove the equality (19). Truly,

$$
B(0)=(-1)^{n} z_{1} z_{2} \ldots . z_{n}=(-1)^{n} R^{n} e^{i\left(\frac{2 \pi}{n}+2 \frac{2 \pi}{n}+\cdots+(n-1) \frac{2 \pi}{n}\right)}=-R^{n} .
$$

Let us verify the validity of equalities (20). To do this, decompose a finite Blaschke product $\mathrm{B}(\mathrm{z})$ into a Maclaurin series. Let

$$
B(z)=a_{0}+a_{1} z+a_{2} z^{2}+\cdots+a_{j} z^{j}+\cdots
$$

From here (see (18)),

$$
B\left(e^{i \frac{2 \pi}{n}} Z\right)=a_{0}+a_{1} e^{i \frac{2 \pi}{n}} Z+\cdots+a_{j}\left(e^{i \frac{2 \pi}{n}}\right)^{j} Z^{j}+\cdots .
$$

If $1 \leq j \leq n-1$, then

$$
e^{i j \frac{2 \pi}{n}} \neq 1
$$

and that means $a_{j}=0$. Since

$$
a_{j}=\frac{B^{(j)}(0)}{j !}
$$

then equalities (20) follow from here.

Note. It is easy to verify that if the number $\mathrm{j}$ is not a multiple of $\mathrm{n}$, then

$$
B^{(j)}(0)=0 \text {. }
$$

Lemma 2. The following relation holds

$$
d=\sup _{f(z) \in H_{p}^{1}}\left|f^{\prime}(0)\right|=\frac{1}{(2 \pi)^{\frac{1}{p}}}
$$

and the extremal function of problem (21) with $p>1$ is unique up to a factor $e^{i \delta}(\delta \in R)$ and has the form

$$
f^{*}(z)=e^{i \delta} \frac{1}{(2 \pi)^{\frac{1}{p}}} Z
$$


When $\mathrm{p}=1$, the extremal function of problem (21) is not unique, and in this case, each of extremal functions $f^{*}(z)$ has the form

$$
f^{*}(z)=e^{i \delta} \frac{1}{2 \pi\left(1+|a|^{2}\right)}(z-a)(1-\bar{a} z),
$$

where $\delta$ - real number $(\delta \in R) ; a$ - any complex number $(|a| \leq 1)$.

Proof. Since the function $F^{*}(z)=\frac{1}{(2 \pi)^{\frac{1}{p}}} z \in H_{p}^{1}$, then

$$
d \geq \frac{1}{(2 \pi)^{\frac{1}{p}}}
$$

with all $1 \leq p<\infty$. After that, we consider two cases.

Let's assume that $p>1$ and $\mathrm{f}(\mathrm{z})$ is any function $\left(f(z) \in H_{p}^{1}\right)$. Then, applying the Hölder's inequality, we get

$$
\begin{gathered}
\left|f^{\prime}(0)\right|=\left|\frac{1}{2 \pi i} \int_{\Gamma} \frac{f(\zeta) d \zeta}{\zeta^{2}}\right| \leq \frac{1}{2 \pi} \int_{\Gamma \mid}|f(\zeta)| d \varphi \leq \frac{1}{2 \pi}\left(\int_{\Gamma}|f(\zeta)|^{p} d \varphi\right)^{\frac{1}{p}}\left(\int_{\Gamma} d \varphi\right)^{\frac{1}{q}} \\
\leq \frac{1}{2 \pi}(2 \pi)^{\frac{1}{q}}=\frac{1}{(2 \pi)^{\frac{1}{p}}} .
\end{gathered}
$$

This implies the equality (21). The extremal function of the problem (21) with $p>1$ is unique up to a constant factor that is equal to one in modulus (see (25) and the introduction) and has the form (22).

Now let's assume that $\mathrm{p}=1$ and $\mathrm{f}(\mathrm{z})$ is any function $\left(f(z) \in H_{1}^{1}\right)$. Then

$$
\left|f^{\prime}(0)\right|=\frac{1}{2 \pi}\left|\int_{\Gamma} \frac{f(\zeta)}{\zeta^{2}} d \zeta\right| \leq \frac{1}{2 \pi} \int_{\Gamma}|f(\zeta)| d \varphi \leq \frac{1}{2 \pi} .
$$

This implies the equality (21) with $\mathrm{p}=1$ (see (24)). In this case, the extremal function of the problem (21), as we will see later, is not unique. Let $f^{*}(z)$ be any extremal function of the problem (21). Let $\varphi^{*}(z)$ be the extremal function of the dual problem (see (5)), in which

$$
\omega(\zeta)=\frac{1}{2 \pi i} \frac{1}{\zeta^{2}}
$$

Then the duality relation connecting extremal functions has the form (see (6))

$$
f^{*}(\zeta)\left[\frac{1}{2 \pi i} \frac{1}{\zeta^{2}}-\varphi^{*}(\zeta)\right] d \zeta=e^{i \delta} \lambda\left|f^{*}(\zeta)\right| d \varphi .
$$

Let's substitute the following functions into relation $(26): f^{*}(\zeta)=\frac{1}{2 \pi}, \varphi^{*}(\zeta)=0, \lambda=$ $\frac{1}{2 \pi}, \mathrm{e}^{\mathrm{i} \delta}=1$. Since $\mathrm{d} \zeta=\mathrm{i} \zeta \mathrm{d} \varphi$, the equality (26) holds. Therefore, $\varphi^{*}(\mathrm{z})=0(\mathrm{z} \in \overline{\mathrm{K}})$. Let's apply the relation (12). We will get

$$
f^{*}(z) \frac{1}{2 \pi i} \frac{1}{z^{2}}=C_{1} \frac{(z-a)(1-\bar{a} z)}{z^{2}},
$$

where $\mathrm{C}_{1}-$ some number. From here

$$
f^{*}(z)=C(z-a)(z-\bar{a} z),
$$

where $C$ - some number; $|a| \leq 1$. Let's find constant number $C$. To do this, we calculate the following integral:

$$
\int_{\Gamma}|\zeta-a||1-\bar{a} \zeta| d \varphi
$$


Since $\zeta=e^{i \varphi}, d \varphi=\frac{d \zeta}{i \zeta}, \bar{\zeta}=\frac{1}{\zeta},|\zeta-a|=|1-\bar{a} \zeta|, \quad$ then $\int_{\Gamma}|\zeta-a||1-\bar{a} \zeta| d \varphi=$ $\int_{\Gamma}|\zeta-a|^{2} d \varphi=\frac{1}{i} \int_{\Gamma}(\zeta-a)(\bar{\zeta}-\bar{a}) \frac{d \zeta}{\zeta}=\frac{1}{i} \int_{\Gamma}(\zeta-a)\left(\frac{1}{\zeta}-\bar{a}\right) \frac{1}{\zeta} d \zeta=$ $\frac{1}{i} \int_{\Gamma} \frac{\zeta-a-\bar{a} \zeta^{2}+|a|^{2} \zeta}{\zeta^{2}} d \zeta$

Denote

$$
\Phi(z)=\frac{z-a-\bar{a} z^{2}+|a|^{2} z}{z^{2}} .
$$

Let's find the residue of this function at zero. We have

$$
\underset{z=0}{\operatorname{res}} \Phi(z)=\lim _{z \rightarrow 0}\left(z^{2} \frac{z-a-\bar{a} z^{2}+|a|^{2} z}{z^{2}}\right)^{\prime}=\lim _{z \rightarrow 0}\left(1-2 \bar{a} z+|a|^{2}\right)=1+|a|^{2} .
$$

From here it follows that

$$
\int_{\Gamma}|\zeta-a||1-\bar{a} \zeta| d \varphi=2 \pi i \frac{1}{i}\left(1+|a|^{2}\right)=2 \pi\left(1+|a|^{2}\right)
$$

It means (see (9))

$$
|C|=\frac{1}{2 \pi\left(1+|a|^{2}\right)} .
$$

Therefore, the extremal function of problem (21) with $\mathrm{p}=1$ is not unique and has the form (23) (see (27)). The lemma is proved.

Theorem 1. The error of the best method for approximating the values of $f^{\prime}(0)$ by the values of $f\left(z_{1}\right), \ldots, f\left(z_{n}\right)$, where $f(z) \in H_{p}$, and the points $z_{1}, \ldots, z_{n}$ are calculated using relations (2), is calculated according to the formula

$$
r_{1, p}\left(0, z_{1}, \ldots, z_{n}\right)=\mathrm{R}^{\mathrm{n}} \frac{1}{(2 \pi)^{\frac{1}{p}}}
$$

for all values $1 \leq \mathrm{p}<\infty$. Moreover, for $\mathrm{p}>1$, the extremal function $\mathrm{f}^{*}(\mathrm{z})$ of the problem (4) is unique up to a constant factor that is equal to one in modulus and has the form

$$
\mathrm{f}^{*}(\mathrm{z})=\mathrm{e}^{\mathrm{i} \delta} \frac{1}{(2 \pi)^{\frac{1}{\mathrm{p}}}} \mathrm{zB}(\mathrm{z})
$$

where $\delta-$ constant real number.

With $\mathrm{p}=1$, the extremal function $\mathrm{f}^{*}(\mathrm{z})$ of the problem (4) is not unique, and each of them has the

$$
\mathrm{f}^{*}(\mathrm{z})=\mathrm{e}^{\mathrm{i} \delta} \frac{1}{2 \pi\left(1+|\mathrm{a}|^{2}\right)}(\mathrm{z}-\mathrm{a})(1-\overline{\mathrm{a}} \mathrm{z}) \mathrm{B}(\mathrm{z})
$$

where $\mathrm{a}$ - any complex number satisfying the condition $|\mathrm{a}| \leq 1 ; \delta \in \mathrm{R}$.

Proof. Let's denote (see (4))

$$
A=\left\{f(z): f(z) \in H_{p}^{1} ; f\left(z_{1}\right)=\cdots=f\left(z_{n}\right)=0\right\}
$$

family of analytic functions. First, we factor the family of functions A. Let $f(z) \in A$ and

$$
g(z)=\frac{f(z)}{B(z)} .
$$

It's clear that $g(z) \in H_{p}^{1}$. This implies that any function $\mathrm{f}(\mathrm{z})(f(z) \in A)$ has the form (19))

$f(z)=B(z) g(z)$, where $g(z) \in H_{p}^{1}$. The reverse is also true. Therefore, (see (20), 


$$
f^{\prime}(0)=B^{\prime}(0) g(0)+B(0) g^{\prime}(0)=B(0) g^{\prime}(0)=-R^{n} g^{\prime}(0) .
$$

Thus, (see (13))

$$
r_{1, p}\left(0, z_{1}, \ldots, z_{n}\right)=R^{n} \sup _{g \in H_{p}^{\prime}}\left|g^{\prime}(0)\right|=R^{n} \frac{1}{(2 \pi)^{\frac{1}{p}}} .
$$

It is clear from the above that for $p>1$, the extremal function $f^{*}(z)$ of the problem (4) has the form (29), and for $\mathrm{p}=1$, it has the form (30)). The lemma is proved.

\subsection{Calculation of the coefficients of the best linear method for approximating the values of $f^{\prime}(0)$ by the values of $f\left(z_{1}\right), \ldots, f\left(z_{n}\right)$}

Lemma 3. If $\sum_{k=1}^{n} c_{k} f\left(z_{k}\right)$ - the best linear method for approximating values of $f^{\prime}(0)$ by the values of $f\left(z_{1}\right), \ldots, f\left(z_{n}\right)\left(f(z) \in H_{p}^{1} ; 1 \leq p<\infty\right)$, then any extremal function $f^{*}(z)$ of the problem $(4)$ is an extremal function of the next problem

$$
\sup _{f \in H_{p}^{1}}\left|f^{\prime}(0)-\sum_{k=1}^{n} c_{k} f\left(z_{k}\right)\right|=r_{1, p}\left(0, z_{1}, \ldots, z_{n}\right) .
$$

Proof. Since $\left|f^{* \prime}(0)\right|=r_{1, p}\left(0, z_{1}, \ldots, z_{n}\right)$ and $f^{*}(z) \in A$ (see (4), (31)), then it follows that $f^{*}(z)$ is the extremal function of the problem (32).

Theorem 2. For any $1 \leq p<\infty$, the best linear approximation method $\sum_{k=1}^{n} c_{k} f\left(z_{k}\right)$ for the values of $f^{\prime}(0)$ by the values of $f\left(z_{1}\right), \ldots, f\left(z_{n}\right) \quad\left(f(z) \in H_{p}\right)$ is unique, and its coefficients are found by the formula

$c_{k}=\frac{1}{R}\left(1-R^{2 n}\right) e^{-i(k-1) \frac{2 \pi}{n}}$,

where $k=1, \ldots, n$.

Proof. Let's consider the following integral

$$
J=\frac{1}{2 \pi i} \int_{\Gamma} \frac{B(0)}{B(\zeta) \zeta^{2}} f(\zeta) d \zeta
$$

where $f(\zeta)$ - any function that satisfies the condition $f(\zeta) \in H_{p}^{1}(1 \leq p<\infty)$. We first estimate this integral modulo from above.

Let $p>1$. We obtain (see (19), (28))

$$
\begin{aligned}
|J| \leq \frac{1}{2 \pi} \int_{\Gamma}\left|\frac{B(0)}{B(\zeta) \zeta^{2}}\right||f(\zeta)| d \varphi & \\
& =\frac{R^{n}}{2 \pi} \int_{\Gamma}|f(\zeta)| d \varphi \leq \frac{R^{n}}{2 \pi}\left(\int_{\Gamma}|f(\zeta)|^{p} d \varphi\right)^{\frac{1}{p}}\left(\int_{\Gamma} d \varphi\right)^{\frac{1}{q}} \leq \frac{R^{n}}{(2 \pi)^{\frac{1}{p}}} \\
& =r_{1, p}\left(0, z_{1}, \ldots z_{n}\right)
\end{aligned}
$$

(here we applied the Hölder's inequality).

Now let $\mathrm{p}=1$. We have (see (19), (28))

$$
|J| \leq \frac{1}{2 \pi} \int_{\Gamma}|B(0)||f(\zeta)| d \varphi \leq \frac{R^{n}}{2 \pi}=r_{1,1}\left(0, \mathrm{z}_{1}, \ldots, \mathrm{z}_{\mathrm{n}}\right) .
$$

Now we calculate the integral $\mathrm{J}$ (applying the basic residue theorem). Denote

$$
\mathrm{Q}(\mathrm{z})=\frac{\mathrm{B}(0)}{\mathrm{B}(\mathrm{z}) \mathrm{z}^{2}} \text {. }
$$


The function $\mathrm{Q}(\mathrm{z})$ has specific points $0, z_{1}, \ldots, z_{n}$ (poles). Therefore, it can be represented as

$$
Q(z)=\frac{c_{-2}}{z^{2}}+\frac{c_{-1}}{z}-\frac{c_{1}}{z-z_{1}}-\cdots-\frac{c_{n}}{z-z_{n}}+\psi(z),
$$

where $\psi(\mathrm{z})$ - function analytic in the K circle; $\mathrm{c}_{-2}, \mathrm{c}_{-1}, \mathrm{c}_{1}, \ldots, \mathrm{c}_{\mathrm{n}}-$ constant numbers. Let's find these numbers. It's clear that (see (38), (37))

$$
\mathrm{C}_{-2}=\lim _{\mathrm{z} \rightarrow 0} \mathrm{z}^{2} \mathrm{Q}(\mathrm{z})=\lim _{\mathrm{z} \rightarrow 0} \frac{\mathrm{B}(0)}{\mathrm{B}(\mathrm{z})}=1 \text {. }
$$

Then (see (20))

$$
\mathrm{c}_{-1}=\underset{\mathrm{z}=0}{\operatorname{res}} \mathrm{Q}(\mathrm{z})=\lim _{\mathrm{z} \rightarrow 0}\left(\mathrm{z}^{2} \frac{\mathrm{B}(0)}{\mathrm{B}(\mathrm{z}) \mathrm{z}^{2}}\right)^{\prime}=-\frac{\mathrm{B}^{\prime}(0)}{\mathrm{B}(0)}=0 .
$$
Since

After that, we find the residues in the simple poles $z_{1}, \ldots, z_{n}$. First, we note the following.

$$
B(z)=\frac{z-R}{1-R z} \prod_{j=2}^{n} \frac{z-z_{j}}{1-\overline{z_{j}} z}
$$

then (see (15), (16))

$$
B^{\prime}(R)=\frac{1}{1-R^{2}} \prod_{j=2}^{n} \frac{R-z_{j}}{1-\bar{z}_{j} R}=\frac{1}{1-R^{2}} \frac{\prod_{j=2}^{n}\left(R-z_{j}\right)}{\prod_{j=2}^{n}\left(1-\bar{z}_{j} R\right)}=\frac{1}{1-R^{2}} \frac{R^{n-1} n}{\left(\frac{1-R^{2 n}}{1-R^{2}}\right)}=\frac{R^{n-1} n}{1-R^{2 n}} .
$$

Further, it follows from the formula (18)

$$
B^{\prime}\left(e^{i \frac{2 \pi}{n}} Z\right)=e^{-i \frac{2 \pi}{n}} B^{\prime}(Z)
$$

From here it follows that

$$
\begin{aligned}
& B^{\prime}\left(z_{2}\right)=e^{-i \frac{2 \pi}{n}} B^{\prime}(R), \\
& B^{\prime}\left(z_{3}\right)=e^{-2 \frac{2 \pi}{n}} B^{\prime}(R) .
\end{aligned}
$$

And in general,

$$
B^{\prime}\left(z_{k}\right)=e^{-i(k-1) \frac{2 \pi}{n}} B^{\prime}(R)
$$

So (see (41)),

$$
B^{\prime}\left(z_{k}\right)=e^{-i(k-1) \frac{2 \pi}{n} \frac{R^{n-1} n}{1-R^{2 n}}}
$$

For all $k=1, \ldots, n$. After that, we calculate the residues of the function $\mathrm{Q}(\mathrm{z})$ at the points $z_{1}, \ldots, z_{n}$. We obtain (see (41), (2), (19))

$$
\begin{aligned}
-c_{k}=\operatorname{res}_{z=z_{k}} Q(z)= & \lim _{z \rightarrow z_{k}}\left(z-z_{k}\right) \frac{B(0)}{B(z) z^{2}}=\frac{B(0)}{B^{\prime}\left(z_{k}\right) z_{k}^{2}}=\frac{-R^{n}\left(1-R^{2 n}\right)}{R^{n-1} n R^{2} e^{i 2(k-1) \frac{2 \pi}{n}}} e^{i(k-1) \frac{2 \pi}{n}} \\
& =-\frac{1}{R}\left(1-R^{2 n}\right) e^{-i(k-1) \frac{2 \pi}{n}} .
\end{aligned}
$$

Therefore,

$$
J=\frac{1}{2 \pi i} \int_{\Gamma}\left(\frac{1}{\zeta^{2}}-\frac{c_{1}}{z-z_{1}}-\cdots+\frac{c_{n}}{z-z_{n}}+\psi(\zeta)\right) f(\zeta) d \zeta=f^{\prime}(0)-\sum_{k=1}^{n} c_{k} f\left(z_{k}\right) .
$$

So, for any function $f(z) \in H_{p}^{1}(1 \leq p<\infty)$, the following inequality holds (see (35)) 


$$
\left|f^{\prime}(0)-\sum_{k=1}^{n} c_{k} f\left(z_{k}\right)\right| \leq r_{1, p}\left(0, z_{1}, \ldots, z_{n}\right) .
$$

Hence the coefficients (see (43))

$$
c_{k}=\frac{1}{R}\left(1-R^{2 n}\right) e^{-i(k-1) \frac{2 \pi}{n}}
$$

are the coefficients of the best linear approximation method; $k=1, \ldots, n$. It remains to verify the uniqueness of the best linear method. Let $f^{*}(z)$ be an extremal function of the problem (4), and $\sum_{k=1}^{n} c_{k} f\left(z_{k}\right)$ - the best linear method (we assume that $p \geq 1$ ). Then (see Lemma 3 )

$$
\sup _{f \in H_{p}^{1}}\left|\int_{\Gamma} \omega(\zeta) f(\zeta) d \zeta\right|=r_{1, p}\left(0, z_{1}, \ldots, z_{n}\right),
$$

where

$$
\omega(\zeta)=\frac{1}{2 \pi i}\left(\frac{1}{\zeta^{2}}-\sum_{k=1}^{n} \frac{c_{k}}{\zeta-z_{k}}\right)
$$

Hence the following relation holds (see (6), (8))

$$
f^{*}(\zeta)\left(\frac{1}{2 \pi i}\left(\frac{1}{\zeta^{2}}-\sum_{k=1}^{n} \frac{c_{k}}{\zeta-z_{k}}\right)-\varphi^{*}(\zeta)\right) d \zeta=e^{i \delta_{1}} \lambda\left|f^{*}(\zeta)\right|^{p} d \varphi,
$$

where $\varphi^{*}(\zeta)$ - the extremal function of the dual problem (see (5), (7)), $\delta_{1}-$ a constant number $\left(\delta_{1} \in R\right)$, and $\lambda-$ the total value in relations (5) or (7). Suppose that there is another best linear method $\sum_{k=1}^{n} \widehat{c_{k}} f\left(z_{k}\right)$. Then the following relation holds

$$
f^{*}(\zeta)\left(\frac{1}{2 \pi i}\left(\frac{1}{\zeta^{2}}-\sum_{k=1}^{n} \frac{\widehat{c_{k}}}{\zeta-z_{k}}\right)-\varphi_{1}^{*}(\zeta)\right) d \zeta=e^{i \delta_{2}} \lambda\left|f^{*}(\zeta)\right|^{p} d \varphi,
$$

where $\varphi_{1}^{*}-$ the extremal function of the dual problem; $\delta_{2}-$ number $\left(\delta_{2} \in R\right), \lambda-$ the total value in relations (5) or (7). Let's consider the function

$$
P(z)=\frac{\frac{1}{2 \pi i}\left(\frac{1}{\zeta^{2}}-\sum_{k=1}^{n} \frac{\widehat{z_{k}}}{\zeta-z_{k}}\right)-\varphi_{1}^{*}(\zeta)}{\frac{1}{2 \pi i}\left(\frac{1}{\zeta^{2}}-\sum_{k=1}^{n} \frac{c_{k}}{\zeta-z_{k}}\right)-\varphi^{*}(\zeta)} .
$$

It is easy to show that

$$
\lim _{z \rightarrow 0} P(z)=1 .
$$

Since $P(\zeta)=e^{i \delta}(\zeta \in \Gamma$ ) (see (44), (45)) and $P(0)=1$, then $P(z)=1, z \in K$ (here $\left.\delta=\delta_{2}-\delta_{1}\right)$. This implies that $\widehat{c_{k}}=c_{k}$ for all $k=1, \ldots, n$. That is, for all $p \geq 1$, the best linear approximation method is unique. The theorem is proved.

\section{Results}

Thus, as a result of solving the problem, the error of the best approximation method is calculated, the corresponding extremal function is found, the uniqueness of the best linear recovery method is proved, and finally, the coefficients of the best linear approximation method are determined. 


\section{Conclusion}

Thus, the problem of optimal recovery of the values of $f^{\prime}(0)$ by the values of $f\left(z_{1}\right), \ldots, f\left(z_{n}\right)$ is solved in the case when the points $z_{1}, \ldots, z_{n}$ form a regular polygon, and the functions $(z) \in$ $H^{p}(1 \leq p<\infty)$. The study can be continued for optimal recovery of the values of $f^{(N)}(0)(N=2,3, \ldots)$ by the values of $f\left(z_{1}\right), \ldots, f\left(z_{n}\right)\left(f(z) \in H^{p}\right)$, where the points $z_{1}, \ldots, z_{n}$ form a regular polygon.

\section{References}

1. C. Micchelli, T. Rivlin, T. Rivlin Lect. Notes 9, 21-93 (1982)

2. T.J. Rivlin, Contemp. Math. 9, 121-151 (1982)

3. S.D. Fisher, C.A. Micchelli, Duke Math. J. 47(4), 789-801(1980)

4. A.B. Pevny, Journal of the Russian Universities. Mathematics 5, 43-49 (1986)

5. K.Yu. Osipenko, M.I. Stesin, Mathematical Notes 49(4), 95-104 (1991)

6. K.Yu. Osipenko, Mathematical Notes 19(1), 29-40 (1976)

7. J. Garnett, Bounded analytic functions (Mir, Moscow, 1984)

8. P. Kusis, Introduction to the theory of $H^{p}$ spaces (Mir, Moscow, 1984)

9. S.Ya. Khavinson, Fundamentals of the theory of extremal problems for bounded analytic functions and their various generalizations (Moscow Institute of Civil Engineering named after V.V. Kuybyshev (MISI), M., 1981)

10. W.W. Rogosinski, H. Schapiro, Acta Math. 90(3), 287-318 (1954)

11. M.P. Ovchintsev, E.M. Gusakova, Vestnik MGSU 4, 44-50 (2014) 